\title{
Addressing age related hearing loss through engineering accessible and affordable hearing technology
}

\author{
Soham Sinha, ${ }^{1}$ Urvaksh D. Irani, ${ }^{2}$ Vinaya Manchaiah, ${ }^{3}$ and M. Saad Bhamla ${ }^{1, *}$ \\ ${ }^{1}$ School of Chemical \& Biomolecular Engineering, \\ Georgia Institute of Technology, 311 Ferst Drive NW, Atlanta, GA 30332, USA \\ ${ }^{2}$ School of Mechanical Engineering, Georgia Institute of Technology, 311 Ferst Drive NW, Atlanta, GA 30332, USA \\ ${ }^{3}$ Department of Speech and Hearing Sciences, Lamar University, \\ $4400 S M L$ King Jr Pkwy, Beaumont, TX 7rry05, USA
}

(Dated: October 20, 2019)

\begin{abstract}
Hearing Aids have dominated the audiological market for decades. While the costs of the electronic components have reduced substantially, the cost of a hearing aid has risen steadily to the point that it has become unaffordable for the vast majority of the population with Age Related Hearing Loss (ARHL). Here, we present an ultra-low-cost, affordable and accessible hearing aid device ('LoCHAid'), specifically targeted for ARHL in elderly patients. The LoCHAid costs only 98 cents $(<\$ 1)$ to mass manufacture and can be personalized for each user through a 3D-printable case. It is designed to be an over-the-counter (OTC) self-serviceable solution for elderly individuals with ARHL. Electroacoustic measurements show that the device meets most of the targets set out by the WHO Preferred Product Profile and Consumer Technology Association for Hearing Aids. The frequency response of the hearing aid shows selectable gain in the range of $4-8 \mathrm{kHz}$, and mild to moderate gain between $200-1000 \mathrm{~Hz}$, and shows very limited total distortion (1\%). Simulated gain measurements show that the LoCHAid is well fitted to a range of ARHL profiles for males and females between the ages of 60-79 years. Overall, the measurements show that the device has the potential to benefit individuals with ARHL. Thus, our proposed design addresses a long-standing and grand challenge of affordable and accessible hearing technology for every elderly person on this planet.
\end{abstract}

\section{INTRODUCTION}

Age Related Hearing Loss (ARHL) is one of the most frequent chronic conditions in older adults with an estimated affected population of 226 million individuals over the age of 65 around the world, which is projected to grow to 900 million by 2050 [1]. Countries in sub-Saharan Africa, South Asia, and Asia Pacific have a prevalence of ARHL that is 4 times higher than in developed nations [1]. The condition is characterised by increasing hearing loss from $1 \mathrm{kHz}$ onwards in the high frequency region $[2,3]$. ARHL results in various physical, mental, and social consequences such as communication difficulties [4-6]. These combined can further exacerbate or cause anxiety, depression, and social isolation, leading to an overall lower health-related quality of life (HRQoL) $[5,7]$. While there is no cure for ARHL, hearing aids are the most frequently used in rehabilitation to improve HRQoL. However, the adoption of hearing aids is very low amongst adults. In low- and middle-income countries (LMIC), hearing aid adoption rates are below $3 \%$ whereas in non-LMIC countries, the adoption rate is around $20 \%$ [1]. Various reasons (e.g., self-reported hearing disability, access to hearing healthcare) may contribute to this poor uptake [8]; however, cost is one of the most substantial factors [8-14]. The retail price of a pair of hearing aids range between $\$ 1,000$ (low-end) to $\$ 8,000$ (highend), with an average price being $\$ 4,700$ in the United States $[7,15]$.

The reasons for the high cost include proprietary soft- ware and hardware, costs of distribution, and failure by public policy such as Medicare and private insurance companies to cover them [7, 9-11]. Even though various low-cost solutions $(<\$ 300)$ have been developed in the last decade such as personal sound amplifiers (PSAPs), they have been reported to have poor acoustic characteristics and often do not meet the acoustic characteristics needed to treat ARHL. They are characterised by having too much low frequency gain and limited high frequency gain, dangerous levels of amplification, excessive internal noise, and high distortion [11, 13, 14, 16-19]. Moreover, over-the-counter (OTC) hearing aids and PSAPs are still between $\$ 100$ and $\$ 500[11,14,17-19]$, which is significantly expensive for people living in LMIC, where the annual healthcare expenditure per capita ranges from $\$ 5$ to $\$ 50$ (2010 USD) [20, 21]. Thus, there is an urgent global need for accessible and affordable hearing devices, potentially served OTC similar to reading glasses, which is further supported by both the World Health Organisation and the U.S. National Academies of Science and Engineering [1, 12, 22].

To address this need, we explore the development of a minimal component hearing aid to address ARHL. We aim to engineer an accessible and affordable minimal device with the required electroacoustic characteristics to benefit elderly users with ARHL. To that extent, we develop a hearing device, coined 'LoCHAid', which costs $\$ 0.98$ when mass produced at 10,000 units. We test the device in laboratory conditions using two methods. First, we test the electroacoustic characteristics in an 
anechoic chamber to examine its properties such as gain, frequency response, harmonic distortion, and equivalent input noise. Second, we simulate the preferred gain for a range of ARHL profiles (SI Fig S1) in both a coupler (Verifit Speechmap), and a real-ear simulator (G.R.A.S KEMAR). We compare the LoCHAid response to these profiles and show that the device provides appropriate gain for a range of average mild to moderate ARHL audiometric patterns, for both males and females (left and right ears) in the age range of 60-79 years.

\section{RESULTS}

\section{LoCHAid as a Modular Device}

The LoCHAid is a modular hearing aid device, which is based primarily on mass manufactured modular components. These include an electret microphone with an automatic gain control and preamplifier, a Class D Stereo Amplifier, a frequency filter, and a standard $3.5 \mathrm{~mm}$ audio jack. The audio jack allows for direct audio output and it allows the use of any closed form sound transducer such as headphones, or earphones. The frequency filter is a second order high pass passive resistor-capacitor (RC) filter with a cutoff frequency of $2340 \mathrm{~Hz}$, which enables shaping the response curve. Peripherals such as an on/off switch, volume control knob (potentiometer), and a power source input are included and shown in Fig $1 \mathrm{c}$ and e. The power source requirement is small (3-5.5 $\mathrm{V}$ ) and can be provided from varied a variety of sources such as rechargeable AAA, AA, coin cell, and rectangular lithium ion batteries as shown in Fig 1b. To protect against noise from the power source, a low-pass DC powerline filter is used. For the most compact version, the lithium ion coin cell battery is used (Fig 1a).

To create the device, the components are soldered on to a custom printer circuit board (Fig 1c,d). The schematic for the board is shown in SI Fig S6. The board requires a few solder points and the entire device can be created in under 30 minutes with a soldering iron (SI Video 1). To compactly hold and protect the LoCHAid, a self-fitting $3 \mathrm{D}$-printed case was constructed from polyamide (Nylon 12) (Fig 1a). The configuration is body-worn with attached headphones. However, the device can also be placed in pockets or worn on the arm (Fig 1f). An end user can turn the device on and off, remove the case, replace batteries, turn the volume control knob, and attach headphones.

The device is designed to be durable. The LoCHAid is drop-proof from $6 \mathrm{ft}$ over repeated impacts $(12 \mathrm{x})$ and water-proof up to $6 \mathrm{~cm}$ of depth for 15 seconds (SI Video 3,4 and Fig S5). It lasts approximately 72 hours continuously with a single cell lithium ion battery, or a maximum of 21 days continuously with 2 AA batteries with an average background sound input of 55-60 dB SPL. The operating temperature range is from $-25^{\circ} \mathrm{C}$ to $65^{\circ} \mathrm{C}$. The lifespan of the device is estimated to be 1.5 years.

LoCHAid does not over-amplify loud sounds. There is an inbuilt safety mechanism if the input sound goes above $110 \mathrm{~dB}$ SPL; the device employs an attack and compression ratio of 1:500, and the sound is compressed to below $110 \mathrm{~dB}$ SPL after a hold time of $30 \mathrm{~ms}$ [23]. As a result of the hold time, small interval sharp sounds such as vehicle horns (100-120 dB SPL) are effectively protected against. To diminish loud continuous sounds such as rock concert music (100-130 dB SPL), a user can reduce the amplification easily using the in-built volume control.

When mass produced at 10,000 units with earphones, a coin-cell battery and a holder, the LoCHAid has a cost of $\$ 0.98$ (Table I). Since the LoCHAid is constructed out of mass produced open source electronics, it does not require specialty made parts. As a result, repairs can be completed by a minimally skilled user with a soldering iron and solder. Moreover, the low cost nature allows LoCHAid to be be replaced very quickly and cheaply if parts are damaged, resulting in a relatively easy-to-use OTC device. A personalisable (and potentially fashionable) custom case can be readily 3D-printed using polymers (Nylon 12 as shown in Fig 1a). However, others materials can be readily used for the case, including acrylic, cardboard, and foam. Given that most hearing aids and PSAPs cost around $\$ 4700$ and $\$ 300$ for a pair respectively, our device shows a reduction of cost by $99.98 \%$.

\section{Electroacoustic Analysis}

The WHO Preferred Product Profile for hearing aid technology in low- and middle-income countries (LMIC) has recommendations for certain electroacoustic parameters [12]. The Consumer Technology Association (CTA) of United States, also established guidelines for electroacoustic parameters for OTC devices in wake of the 2017 FDA Reauthorisation Act [24, 25]. These parameters are OSPL 90, OSPL 60, Range of Frequency Response, Total Harmonic Distortion at 500, 1000, $1600 \mathrm{~Hz}$ at $70 \mathrm{~dB}$ SPL input, Equivalent Input Noise (EIN), and High Frequency Average $(1,1.5,3 \mathrm{kHz})$. The values for LoCHAid were benchmarked by using an AudioScan Verifit (version 3.1; AudioScan, Dorchester, ON, Canada) machine that tested the aforementioned parameters in accordance with the ANSI (American National Standards Institute)/ASA S3.22-2014 standards (Fig 2a-c) [26]. Table II compares the parameters of LoCHAid, against WHO Recommendations and CTA level. The frequency response curves for the LoCHAid are shown in Fig 2d.

The overall average gain for the frequency response curve is $15 \mathrm{~dB}$ SPL. The total harmonic distortion is very low at $1 \%$, much less than the limits posed by WHO $(8 \%)$, and CTA (5\%). The device itself has low interference 
with signal integrity, which is a necessary requirement for understanding speech accurately. The maximum OSPL 90 is much higher than the OSPL $90 @ 1$ kHz, which denote that the frequency response is skewed towards one end of the spectrum. Observing the high frequency averages (HFA), we see that the HFA $(4,5,6 \mathrm{kHz})$ @ OSPL90 is $10 \mathrm{~dB}$ SPL higher than the HFA $(1,1.5,3$ $\mathrm{kHz}$ ), which shows that the skewness of the response is directed towards high frequencies. The curves shown in Fig $2 \mathrm{~d}$ highlight that the device is more selectable for high frequencies $(>2 \mathrm{kHz})$, and less selectable $(<1 \mathrm{kHz})$ for low frequencies. This selectivity towards high frequencies is necessary to treat ARHL, as hearing loss increases with frequency (SI Fig S1). The EIN of the device is $10 \mathrm{~dB}$ SPL higher than recommended from WHO PPP and CTA; however, we discuss the implications of this in discussion section below (also see SI Section IV). Overall, we successfully meet 5 out of 6 criteria as set out by WHO PPP and CTA [12].

\section{Simulated Gain Measurements Against ARHL Profiles}

Coupler Gain Simulations Using the Speechmap Test

After examining the electroacoustic characteristics of the LoCHAid, we explore how closely its gain measurements match a range of ARHL audiometric profiles. We compiled a total of 11 clinically averaged ARHL profiles based on age, gender, ear, and severity from previous work $(1994-2004,2008)[2,3]$. These profiles are males and females between the ages of 60-69 for both left and right ears, males and females between the ages of 70 79 for both left and right ears, and three gender neutral ARHL profiles of increasing severity of ARHL denoted by $\mathrm{X}$ (mild), Y (moderate), and Z (severe). The clinically averaged profiles were taken from a total sample size of $\mathrm{N}=1546$ Females, 1345 Males that exhibit ARHL in the United States (SI Fig S1).

Speechmap measurements help show how closely the gain of the hearing aid at different frequencies matches the estimated gain required for ARHL audiometric profiles. The estimated gain for different audiometric patterns at different frequencies is governed by different hearing aid fitting algorithms. We chose the NA-NL2 method, the current industry standard, which takes into account gender, age, and language [27]. The frequency targets are generated at 250, 500, 750, 1000, 1500, 2000, $3000,4000,6000,8000 \mathrm{~Hz}$, giving a total of 10 frequency targets.

Speechmap undertakes this simulation of NA-NL2 targets based on a International Speech Test Signal (ISTS). ISTS is a mixed audio signal representing average speech at different frequencies and languages [28]. Three input sound levels for the signal were considered: $55 \mathrm{~dB}$ SPL (whispering level), $65 \mathrm{~dB}$ SPL (conversational level), and $80 \mathrm{~dB}$ SPL (loud level).

The response of the LoCHAID with full open volume against the targets of all 11 profiles are shown in Fig 3 . To determine goodness of fit, we adopted a Strict and Loose Criteria that has been used previously by other researchers $[11,17,19,29,30]$. If the response of the device is within $5 \mathrm{~dB}$ SPL of the target, then it fits under Strict Criteria, while a response within $10 \mathrm{db}$ SPL is used for the Loose Criteria. Under the Strict Criteria, all 11 profiles match only $10 \%$ of the targets, and $64 \%$ of the profiles match $50 \%$ of the targets. Under the Loose Criteria, $64 \%$ of the profiles match $90 \%$ of the targets, and all 11 profiles match $50 \%$ of the targets. The results reveal that the LoCHAid is a good fit to most profiles. However, not all the profiles are fitted equally well and the response of the device is too high to fit the milder ARHL profiles, such as Females in the 60-69 age range. To better fit the milder profiles, our data suggests to use the LoCHAid at a lower volume setting ( -5 to $-10 \mathrm{~dB}$ SPL). The reader is referred to SI Fig S2 for quantification of fits for each profile, SI Figs S7-S77 and Tables I-LX for individual profile targets and responses.

\section{Real Ear Gain Simulation Using the G.R.A.S KEMAR Manikin}

AudioScan, although reliable, measures the gain via a 0.4 cc wideband coupler, and is not the best representation of a real human ear. Hence, to obtain a more accurate and precise measurement of a real ear, we used a G.R.A.S Knowles Electronics Manikin for Acoustic Research (KEMAR). This manikin is designed to anatomically resemble a real human ear as close as possible, and hence provides a real ear simulation. The device was attached to the KEMAR manikin as shown in the inset of Fig $4 \mathrm{a}$. The ear buds were placed into the ears, and a ISTS signal of strength $65 \mathrm{~dB}$ SPL was played.

Fig 4a details the targets and response for Males 60-69 Left and Right Ears. Under the Strict and Loose Criteria, $70 \%$ and $90 \%$ of the targets are matched, respectively, indicating an overall good fit for this ARHL audiogram. The results for all 11 ARHL profiles are shown in Fig 4b. Under the Strict Criteria, all 11 profiles match $50 \%$ of the targets, and $64 \%$ of the profiles match $70 \%$ of the targets. Based on Loose Criteria, $70 \%$ of all the profiles match $90 \%$ of targets, and all the profiles match $80 \%$ of the targets. The improvement in Strict Criteria matched targets from Speechmap to KEMAR for all the profiles is from $10 \%$ to $50 \%$, and the improvement for Loose Criteria is from $50 \%$ to $80 \%$. Both these improvements show that the device is very well fitted to the profiles. We note that $50 \%$ of all missed targets lie at low frequencies (250 $\mathrm{Hz}, 500 \mathrm{~Hz}$ ) as the device shows very low gain at low frequencies $(<750 \mathrm{~Hz})$. This is desirable as many indi- 
viduals with ARHL often tend to report hearing echo of their own voice, and also hearing low noise such as 'refrigerator noise and humm' (100-200 Hz), which can be distracting [16]. The reader is referred to SI Fig S3 for quantification of fits for each profile, SI Figs S7-S77 and Tables I-LX for individual profile targets and responses.

\section{DISCUSSION}

We designed the LoCHAid to be as affordable as possible. A WHO guideline states that a hearing aid should be no more than $3 \%$ of the gross national product, per capita, per hearing aid [31]. Using current World Bank Figures, a hearing aid in order to be affordable has to be within $\$ 1614$ for United States, $\$ 62$ for India, $\$ 10$ for Ethiopia [32]. For low-income, lower-middle income, and low and middle income countries, the affordable price is $\$ 20, \$ 67.77, \$ 135$, respectively. Our device clearly meets this criteria [32]. Additionally, the LoCHAid is at most $20 \%$ per capita annual health expenditure $(\$ 5-\$ 50)$ for LMIC [20, 21]. For upper middle- to high-income countries, it is less than $1 \%$ of annual health care expenditure per capita $(\$ 1000-\$ 3,000)[20,21]$. We have accomplished this by leveraging off-the-shelf components, mass produced electronics, and printed circuit boards. The lack of specialised electronic components such as digital sound processors and wires all help to not only make the device affordable, but also minimal.

We have designed our device to be accessible for elderly individuals with mild to moderate ARHL. The device is body-worn rather than behind-the-ear (BTE) or in-theear (ITE). The design is geriatric friendly; many elderly patients have trouble handling the small in-the-ear, and BTE and ITE hearing aids, especially those with limited dexterity as a condition of arthritis [17, 33, 34]. The larger model reduces the likelihood of elderly patients misplacing the device, and facilitates the use of slightly larger domestic lithium-ion batteries. Since batteries are an additional cost, we opted to use lithium-ion batteries. Lithium-ion batteries enable longer usage times than zinc-ion batteries, and do not require trips to costly specialised battery markets, which do not exist in LMIC $[31,35,36]$. Previously the cost burden of batteries has been notably addressed by solar technologies such as solar panel rechargers by groups such as Solar Ear [37]. We note that our design is indeed compatible with this philosophy and an off-the-shelf solar charger can be readily employed to charge the lithium ion battery as shown in SI Fig S4. The combined cost for the solar panel, adapter, lithium-ion battery, and LoCHAid is still only $\$ 26.22$ which is still a factor of 4 less than the Solar Ear kit which costs $\$ 100$ [38]. Thus, the hidden annual cost burden of non-rechargeable batteries is also reduced.

We have made the manufacturing and distribution of the LoCHAid accessible as well. Currently, the distri- bution methods of hearing aids are not direct to consumer $[8,31,34]$. Most hearing aids are sold by specialists who are typically audiologists; ear, nose, and throat physicians; and licensed hearing-aid specialists [8, 34]. Practices such as bundling, limited selection of devices, and vertical integration of independent audiological clinics by hearing aid companies, have created barriers to access [34]. Our device circumvents the need for specialised dispensers through its minimalist design, which can be marketed through OTC. We have not only made the device OTC, but also do-it-yourself. The current $\mathrm{PCB}$ configuration is through-hole as it is the easiest to solder upon when manufacturing. Like the open-source Arduino community, our open-source device empowers local communities to be involved in every step of use of the device, from its screening for potential hearing loss, to creation, repair, and disbursement of devices to those in need. Effectively, the open-source nature of LoCHAid makes it accessible for communities to create their own supply chain logistics, which was not addressed in previous work in hearing aids for LMIC (\$140) [39]. Such an approach to combine appropriate technology with a local support base is essential to meet the needs of LMIC, as there is a chronic shortage of trained support personnel for hearing aids [31, 40]

The LoCHAid represents an opportunity to change the value proposition of hearing aids. In European countries such as the United Kingdom, where hearing aids are fully or partially covered under governmental health programs such as National Health Service, uptake remains low at $30 \%$ too $[8,13,34]$. Social stigma is one of the barriers; however, that may be changing with the arrival of an aging population that has grown more comfortable with technology and have a desire for more fashionable, robust, and better hearing technology [1,34]. With LoCHAid, individualisation of the device is just a matter of time. Like owning different pairs of glasses, one can create different 3D-printed casings and designs make it fashionable to one's desire. It creates an opportunity to induce a perception shift, where hearing aids are not seen as a hindrance, but an extension of ones personality.

The electroacoustic analysis shows that the LoCHAid has high frequency gain necessary for ARHL and meets most of the preferred product profile for hearing aid technology suitable for LMIC set out by the WHO. The one characteristic that is deviant from the standard is the EIN. Other researchers have noted that EIN is a measure that is most frequently out of specifications [41, 42]. In a recent study, four most widely used hearing aid models were tested which had an average EIN between 27 to $34.5 \mathrm{~dB}$ SPL [42]. Thus, we anticipate that the relatively high EIN of $40 \mathrm{~dB}$ SPL may hinder speech perception in some users, especially those with relatively mild hearing loss. The EIN can be reduced in future versions of the LoCHAid, potentially with an increased cost. However, we note that the simulated gain measurements of the 
LoCHAid reveal that the device has the necessary gain to provide appropriate amplification for a range of ARHL demographic profiles across a two decade age difference (60-79 years). Although the lab measurements presented here are a first step, further translation and clinical work is necessary to evaluate the individual benefits and outcomes provided by the LoCHAid device.

Ultimately, we show that it is possible to design an ultra-low-cost accessible and affordable OTC open-source hearing aid device that can address mild to moderate ARHL. In the United States, hearing technology regulations are being reconsidered in the wake of the FDA Reauthorisation Act of 2017 [15]; our device is perhaps in the right time period to enact change. Beyond the United States, in LMIC, where the need and growing burden of ARHL is a serious concern, the LoCHAid offers an opportunity to indeed bring 'hearing to the masses' [43].

\section{METHODS}

\section{Construction of LoCHAid}

The LoCHAid was constructed using a handheld soldering iron (X-Tronic Model 3020-XTS Digital Display Soldering Iron Station) with solder (WYCTIN $1.0 \mathrm{~mm}$ 50G 60/40 (Tin-60\% Lead-40\%) Tin Lead Roll 1.8\% Flux Soldering Wire Reel). Foam (EVA Straight Edge Foam) was obtained for ease of construction for the microphone placement, but can be removed after construction. (SI Video 1) The case was designed in SolidWorks v27, and was 3D-printed (Stratasys J750) from blue polyamide (Nylon 12). The electret microphone utilisng MAX 9814, class D stereo amplifier utilising MAX 98306, audio jack, coin cell holder, and 3V coin cell battery was obtained from Adafruit (www.adafruit.com, $\mathrm{P} / \mathrm{N}$ 1713, 987, 1699, 1870, 2849, respectively). The $5 \mathrm{k} \Omega$ resistor, $1 \mathrm{uF}$ capacitor, $6.8 \mathrm{k} \Omega$ resistor, $1000 \mathrm{pF}$ capacitor, $15 \mathrm{uF}$ capacitor, 6 pronged on/off slide switch, and was obtained from Digikey (www.digikey.com, P/N CT6EP502, C0805C105J4RACTU, RMCF0805FT6K80, CL21B102KBANNNC, C1210C156K8PACTU, JS202011CQN, respectively). The potentiometer which provides a volume control of $(+/-10 \mathrm{~dB}$ SPL) was obtained from Amazon (www.amazon.com, $\mathrm{P} / \mathrm{N}$ MCIGICM Potentiometer Breadboard Kit with Knob). The circuit board was printed at Oshpark board printing services (www.oshpark.com). One of the filters is a second order high pass RC filter with a cutoff frequency of $2340 \mathrm{~Hz}$ (constructed of $26.8 \mathrm{k} \Omega$, and $21000 \mathrm{pF}$ capacitors). The other is a low pass powerline filter to subdue noise from the power source. The power source range is $3 \mathrm{~V}-5.5 \mathrm{~V}$. The schematic is shown in SI Fig S6.

\section{Electroacoustic Analysis}

Electroacoustic measurements were performed using the AudioScan Verifit device (version 3.1; AudioScan, Dorchester, ON, Canada). For all tests, a pair of Panasonic RP-HJE125E Wired Earphones - Wired, Orange (RP-HJE125-D) was used. The earbuds' soft plastic bud was removed, and the exposed end was placed into the center of a HA-1 0.2 cc-coupler. Putty (Scotch Lightweight Mounting Putty, 2 oz) was used to seal the coupler, and any other sound openings of the earbud itself outside the coupler. The device was placed inside the anechoic chamber of the machine. The other earbud was sealed off to prevent feedback (SI Video 2). The AudioScan speaker was placed within $2 \mathrm{~mm}$ of the microphone of the device. The entire chamber was completely closed, and the tests were run. The measurements obtained from the LoCHAid were compared against two hearing aid standards, including: (a) WHO preferred product profile for hearing aid technology suitable for LMIC [12]; (b) ANSI S3.222014/CTA-2051 standards for OTC devices [24, 25]. However, considering that the device is primarily aimed towards ARHL individuals in LMIC, the WHO specifications were used for most of the comparisons. The measurements included: output sound pressure level-90 (OSPL-90) curves, high-frequency average full-on gain (HFA FOG), frequency response curves, equivalent input noise (EIN), and total harmonic distortion (THD).

All tests were run with 3 different devices, $\mathrm{N}=3$, with $\mathrm{n}=15$ trials per device.

\section{Simulated Gain Measurements Against ARHL Profiles}

The preferred gain for a range of mild- to moderate ARHL profiles (see SI Fig S1) were simulated and were compared the LoCHAid response to these profiles to check if the device provides appropriate gain for certain ARHL audiometric patterns. The simulated gain measurements were performed using two different methods, which included: (a) Speechmap testing simulating hearing aid gain in a coupler; and (b) simulation in an ear simulator using the KEMAR manikin. The type and extent of ARHL varies across age, ear, and gender. Hence, a range of ARHL profiles was taken from published studies $[2,3]$ and the preferred gain was estimated using the NAL-NL2 prescriptive formula for these profiles. The speechmap and also KEMAR ear simulated measurements of the LoCHAid were compared against these preferred estimated gains. This comparison was to determine whether the LoCHAid could provide appropriate levels of amplification (within 5 or $10 \mathrm{~dB}$ SPL) at 10 frequencies $(250,500,750,1000,1500,2000,3000,4000$, $6000,8000 \mathrm{~Hz})$. 
The Speechmap test was performed using the AudioScan Verifit device. The 0.2 cc-coupler was switched out with a $0.4 \mathrm{cc}$ wideband coupler; the same procedure was followed with removing soft plastic earbuds, and placing the bare plastic part in the middle of the coupler, and sealing the entering side of the coupler. Other holes were also sealed off. The entire chamber was closed and then the tests were run using the ISTS (International Speech Test Signal). ISTS is an internationally recognized test signal that may be used in the technical evaluation of hearing instruments, and for probe-microphone measurements [28]. The ISTS is shaped according to the LTASS (Long Term Average Speech Spectrum) standards. Three test signal strengths were run at $55 \mathrm{~dB}$ SPL (soft/whispering), $65 \mathrm{~dB}$ SPL (average/conversational), and $80 \mathrm{~dB}$ SPL (loud/outside). All tests were run with $\mathrm{N}=3$ devices, $\mathrm{n}=15$ trials overall.

The simulated real-ear feedback measurements were conducted in the G.R.A.S KEMAR manikins left ear. The tests were conducted in an audiological soundproof room with the manikin being inside. The LoCHAid was clipped to the front of the manikin's shirt. The earphones (Panasonic RP-HJE125E Wired Earphones) were placed inside the manikin's ears with the soft plastic buds attached. The loudspeaker was located at an azimuth of 45 degrees and $30 \mathrm{~cm}$ (1 foot) from KEMAR. The center of the loudspeaker was at the same level as the midpoint of the hearing aid. To simulate a normal conversational situation, the input signal used was ISTS at $65 \mathrm{~dB}$ SPL. A single device was tested by playing the exact 40 seconds of the recording. The experimental setup was re-calibrated after every run to make sure that the intensity of the incoming sound was still at $65 \mathrm{~dB}$ SPL, and earbuds if they slipped out were placed back in the ears. The test was run with $\mathrm{N}=1$ device, $\mathrm{n}=15$ trials.

\section{REFERENCES}

* Please address correspondence to: M.S.B (saadb@chbe.gatech.edu)

[1] World Health Organisation, "Addressing the rising prevalence of hearing loss," World Health Organisation (2018).

[2] Lindsay Ciletti and Gregory A. Flamme, "Prevalence of hearing impairment by gender and audiometric configuration: Results from the National Health and Nutrition Examination Survey (1999-2004) and the Keokuk County Rural Health Study (1994-1998)," Journal of the American Academy of Audiology 19, 672-685 (2008).

[3] Karen J. Cruickshanks, Terry L. Wiley, Theodore S. Tweed, Barbara E.K. Klein, Ronald Klein, Julie A. Mares-Perlman, and David M. Nondahl, "Prevalence of hearing loss in older adults in Beaver dam, Wiscon- sin. The epidemiology of hearing loss study," American Journal of Epidemiology 148, 879-886 (1998).

[4] Sharon G Kujawa and M Charles Liberman, "Acceleration of age-related hearing loss by early noise exposure: evidence of a misspent youth." The Journal of neuroscience : the official journal of the Society for Neuroscience 26, 2115-2123 (2006).

[5] Ee-Munn Chia, Jie Jin Wang, Elena Rochtchina, Robert R Cumming, Philip Newall, and Paul Mitchell, "Hearing Impairment and Health-Related Quality of Life: The Blue Mountains Hearing Study," Ear and Hearing 28 (2007).

[6] Niels Christian Stenklev and Einar Laukli, "Presbyacusis - Hearing thresholds and the ISO 7029," International Journal of Audiology 43, 295-306 (2004).

[7] Jan Blustein and Barbara E Weinstein, "Opening the Market for Lower Cost Hearing Aids : Regulatory Change Can Improve the Health of Older Americans," 106, 1032-1036 (2016).

[8] Line Vestergaard Knudsen, Marie Öberg, Claus Nielsen, Graham Naylor, and Sophia E. Kramer, "Factors Influencing Help Seeking, Hearing Aid Uptake, Hearing Aid Use and Satisfaction With Hearing Aids: A Review of the Literature," Trends in Amplification 14, 127-154 (2010).

[9] Abby McCormack and Heather Fortnum, "Why do people fitted with hearing aids not wear them?" International journal of audiology 52, 360-368 (2013).

[10] Anna Marie Jilla, Carole E Johnson, and Jeffrey L Danhauer, "Disruptive Hearing Technologies and Mild Sensorineural Hearing Loss I: Accessibility and Affordability Issues," Seminars in hearing 39, 135-145 (2018).

[11] Bradley McPherson and E. T.L. Wong, "Effectiveness of an affordable hearing aid with elderly persons," Disability and Rehabilitation 27, 601-609 (2005).

[12] World Health Organisation, "Preferred profile for hearing-aid technology suitable for low- and middleincome countries," World Health Organisation (2017).

[13] Vinaya Manchaiah, Brian Taylor, Ashley L. Dockens, Nicole R. Tran, Kayla Lane, Mariana Castle, and Vibhu Grover, "Applications of direct-to-consumer hearing devices for adults with hearing loss: A review," Clinical Interventions in Aging 12, 859-871 (2017).

[14] Vinaya Manchaiah, "Direct-to-Consumer Hearing Devices for Adults With Hearing Loss: Definitions, Summary of Literature, and Analysis of Risks and Benefits," Perspectives of the ASHA Special Interest Groups 3, 511 (2018).

[15] Frank R. Lin, William R. Hazzard, and Dan G. Blazer, "Priorities for improving hearing health care for adults: A report from the national academies of sciences, engineering, and medicine," JAMA - Journal of the American Medical Association 316, 819-820 (2016).

[16] Ricketts Todd A., Dittberner Andrew B., and Johnson Earl E., "High-Frequency Amplification and Sound Quality in Listeners With Normal Through Moderate Hearing Loss," Journal of Speech, Language, and Hearing Research 51, 160-172 (2008).

[17] Zoe Yee Ting Chan and Bradley McPherson, "Over-the-counter hearing aids: A lost decade for change," BioMed Research International 2015 (2015), $10.1155 / 2015 / 827463$.

[18] Chi Man Cheng and Bradley McPherson, "Over-thecounter hearing aids: Electroacoustic characteristics and 
possible target client groups," International Journal of Audiology 39, 110-116 (2000).

[19] Nicholas S. Reed, Joshua Betz, Nicole Kendig, Margaret Korczak, and Frank R. Lin, "Personal sound amplification products vs a conventional hearing aid for speech understanding in noise," JAMA - Journal of the American Medical Association 318, 89-90 (2017).

[20] Ke Xu, Agnes Soucat, Joseph Kutzin, Callum Brindley, Nathalie Vande Maele, Hapsatou Touré, Maria Aranguren Garcia, Dongxue Li, Hélène Barroy, Gabriela Flores, Tomas Roubal, Chandika Indikadahena, Veneta Cherilova, and Andrew Siroka, "Public Spending on Health: A Closer Look at Global Trends:Geneva: World Health Organization," World Health Organization (2018).

[21] United Nations Development Programme, "Global multidimensional poverty index 2019: Illuminating Inequalities," United Nations (2019).

[22] Dan G. Blazer, Sarah Domnitz, and Catharyn T. Liverman, "Hearing health care for adults: Priorities for improving access and affordability," Hearing Health Care for Adults: Priorities for Improving Access and Affordability, , 1-324 (2016).

[23] Maxim Integrated Products, "Microphone Amplifier with AGC and Low-Noise Microphone Bias MAX9814 Microphone Amplifier with AGC and Low-Noise Microphone Bias: Data Sheet," Maxim Integrated (2016).

[24] Consumer Technological Association, "ANSI/CTA Standards-2051," Consumer Technological Association (2017).

[25] Congress of The United States of America, "Food and Drug Administration Reauthorisation Act of 2017," An Act of Congress (2017).

[26] American National Standards Institute, "Specification of Hearing Aid Characteristics," American National Standards 552 (2014).

[27] G. Keidser, H.R. Dillon, M. Flax, T. Ching, and S. Brewer, "The NAL-NL2 prescription procedure," Audiology Research 1, 2010-2012 (2011).

[28] Inga Holube, Stefan Fredelake, Marcel Vlaming, and Birger Kollmeier, "Development and analysis of an international speech test signal (ISTS)," International Journal of Audiology 49, 891-903 (2010).

[29] Hashir Aazh, Brian C. J. Moore, and Deepak Prasher, "The accuracy of matching target insertion gains with open-fit hearing aids," American Journal of Audiology 21, 175-180 (2012).

[30] Hashir Aazh and Brian C.J. Moore, "The value of routine real ear measurement of the gain of digital hearing aids," Journal of the American Academy of Audiology 18, 653664 (2007).

[31] Bradley McPherson, "Innovative Technology in Hearing Instruments: Matching Needs in the Developing World," Trends in Amplification 15, 209-214 (2011).

[32] World Bank, "Gross National Income per Capita (Constant 2010 USD)," (2019).

[33] Mary Bliss, "Use and ownership of hearing aids in elderly people," Lancet 360, 1333-1334 (2002).

[34] President's Council of Advisors on Science and Technology, "Letter to president on aging america hearing loss: Imperative of improved hearing technologies," (2015).

[35] Aroa R. Mainar, Elena Iruin, Luis C. Colmenares, Andriy Kvasha, Iratxe de Meatza, Miguel Bengoechea, Olatz Leonet, Iker Boyano, Zhengcheng Zhang, and J. Alberto
Blazquez, "An overview of progress in electrolytes for secondary zinc-air batteries and other storage systems based on zinc," Journal of Energy Storage 15, 304-328 (2018).

[36] Bradley Mcpherson and Ron Brouillette, "A Fair Hearing for All: Providing Appropriate Amplification in Developing Countries," Communication Disorders Quarterly 25, 219-223 (2004).

[37] Howard Weinstein, "Solar charger for hearing aid," Compendium of innovative health technologies for lowresource settings (2012).

[38] Maria Belenky, "Solar ear: Low-cost hearing aids, solar battery chargers," (2011).

[39] Ricardo Ferreira Bento and Silvio Pires Penteado, "Designing of a Digital Behind-the-Ear Hearing Aid to Meet the World Health Organization Requirements," Trends in Amplification 14, 64-72 (2010).

[40] Bradley McPherson, "Hearing assistive technologies in developing countries: Background, achievements and challenges," Disability and Rehabilitation: Assistive Technology 9, 360-364 (2014).

[41] James D. Lewis, Shawn S. Goodman, and Ruth A. Bentler, "Measurement of hearing aid internal noise," The Journal of the Acoustical Society of America 127, 2521-2528 (2010).

[42] Jourdan T Holder, Erin M Picou, Jill M Gruenwald, and Todd A Ricketts, "Do Modern Hearing Aids Meet ANSI Standards ?" Journal of the American Academy of Audiology 27, 619-627 (2016).

[43] Charles J. Limb, "Hearing for the Masses," Trends in Amplification 14, 63 (2010).

\section{ACKNOWLEDGEMENTS.}

We would like to thank A. Dubey and S. Sekhar for LoCHAid designs; Dr. A. Dockens, Dr. E. Burns of Lamar Audiology for assistance in audiological testing; Dr. R. A. Robinson Jr at the Georgia Tech School of Electrical and Computer Engineering guidance on filter design and frequency response; J. Eng for assistance on design of the custom PCB; Dr. P. S. Russo at Georgia Tech School of Materials Science and Engineering for test wearing the device; and finally the Bhamla Lab for invaluable feedback. M.S.B. acknowledges funding support from Capita Foundation (2018 CFAR award).

\section{AUTHOR CONTRIBUTIONS.}

S.S. and M.S.B concieved idea for device. S.S. constructed device. V.M and S.S conducted audiological tests and analysed data. S.S and U.I designed the external casing and conducted different filter tests. All authors wrote the manuscript.

\section{COMPETING INTERESTS.}

The authors declare no competing financial interests. 
bioRxiv preprint doi: https://doi.org/10.1101/811828; this version posted October 21,2019 . The copyright holder for this preprint (which was not certified by peer review) is the author/funder, who has granted bioRxiv a license to display the preprint in perpetuity. It is made available under aCC-BY-NC-ND 4.0 International license.

\section{DATA AVAILABILITY}

The authors maintain that all data is available and can be found in Github link. 
bioRxiv preprint doi: https://doi.org/10.1101/811828; this version posted October 21,2019 . The copyright holder for this preprint (which was not certified by peer review) is the author/funder, who has granted bioRxiv a license to display the preprint in perpetuity. It is made available under aCC-BY-NC-ND 4.0 International license.
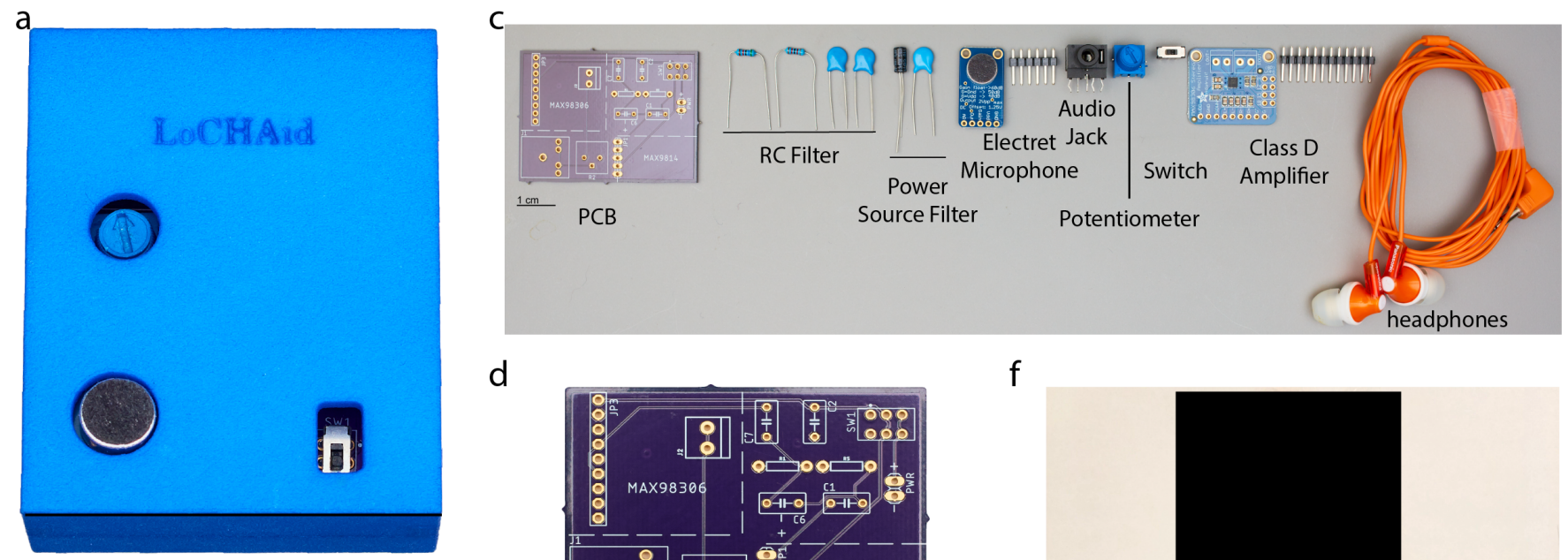

d

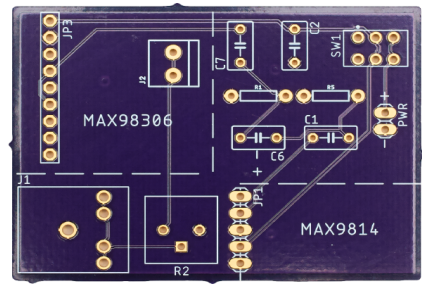

$1 \mathrm{~cm}$

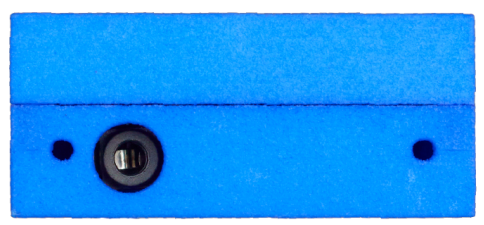

b

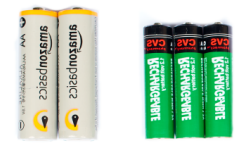

Alkaline AA

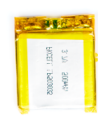

Lithium Ion Coin Cell
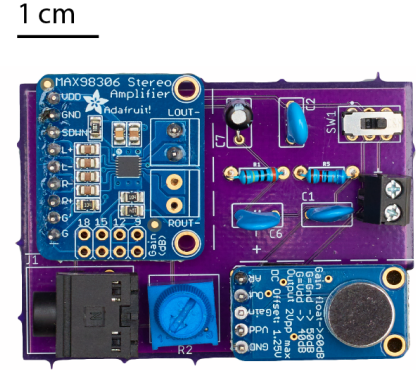

$1 \mathrm{~cm}$ f

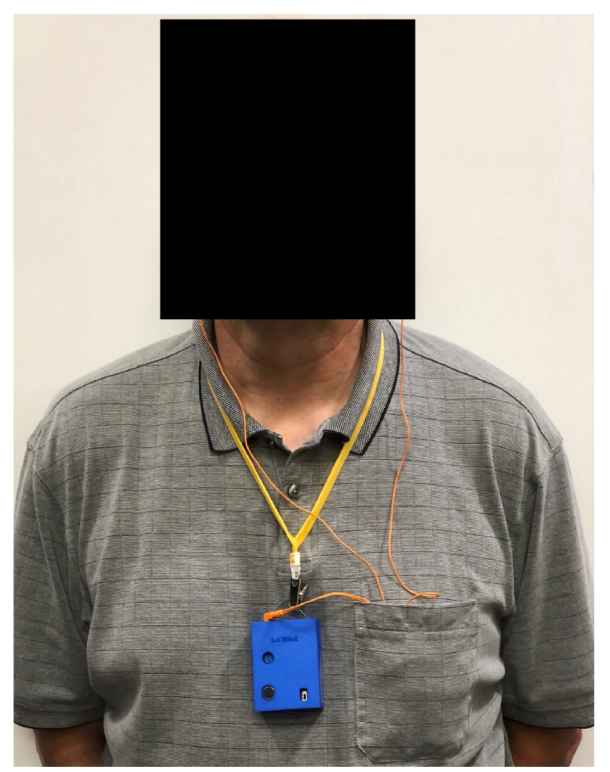

FIG. 1. Construction and Components of the LoCHAid. a. The LoCHAid is shown in its top view, with its 3D printed polyamide (Nylon 12) case tilted. The side view of the audio jack opening and holes for attaching material for neck wear are shown below. The LoCHAid in its case has a size of 2.64 inches by 2.24 inches. The audio jack can incorporate any standard $8 \mathrm{~mm}$ sound transducer. b. Displays various types of batteries such as AA, rechargeable AAA, Lithium Ion flat pack, as well as lithium ion coin cell that can be used to power the device. The device has a power requirement that is between 3-5.5 $\mathrm{V}$. The amount of batteries denote the the number required to power the device. c. The required parts to assemble the device are shown here with group labels; specific details are given in Table I. d. View of the custom printed circuit board (PCB) without any components. e. View of the PCB with components soldered on. f. View of the body-worn device by an anonymous 65 year old male as part of the intended audience of the device. 
bioRxiv preprint doi: https://doi.org/10.1101/811828; this version posted October 21, 2019. The copyright holder for this preprint (which was not certified by peer review) is the author/funder, who has granted bioRxiv a license to display the preprint in perpetuity. It is made available under aCC-BY-NC-ND 4.0 International license.

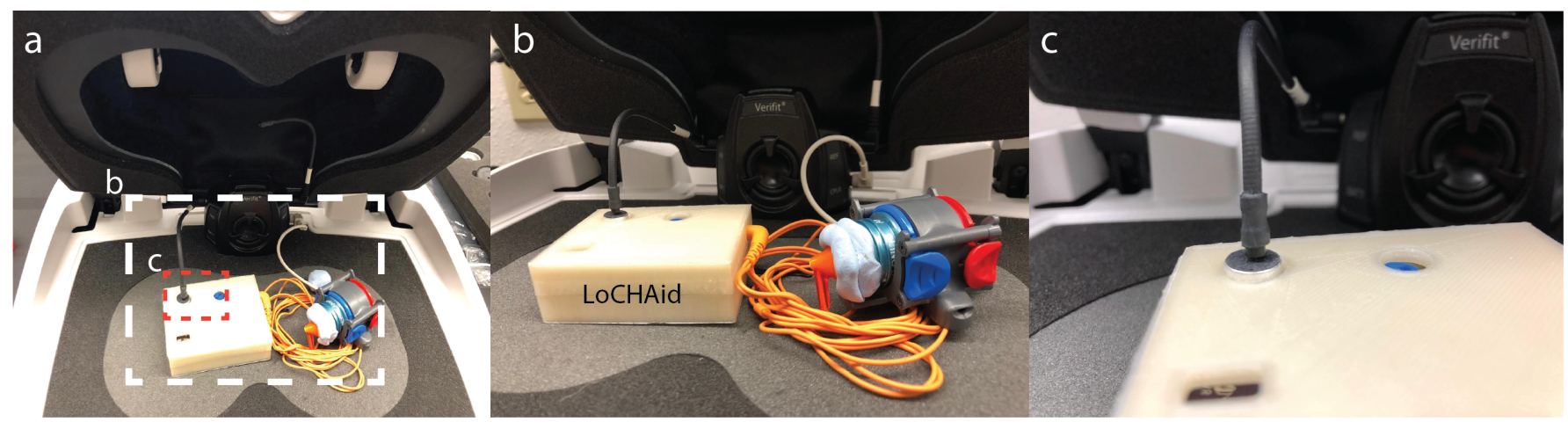

d

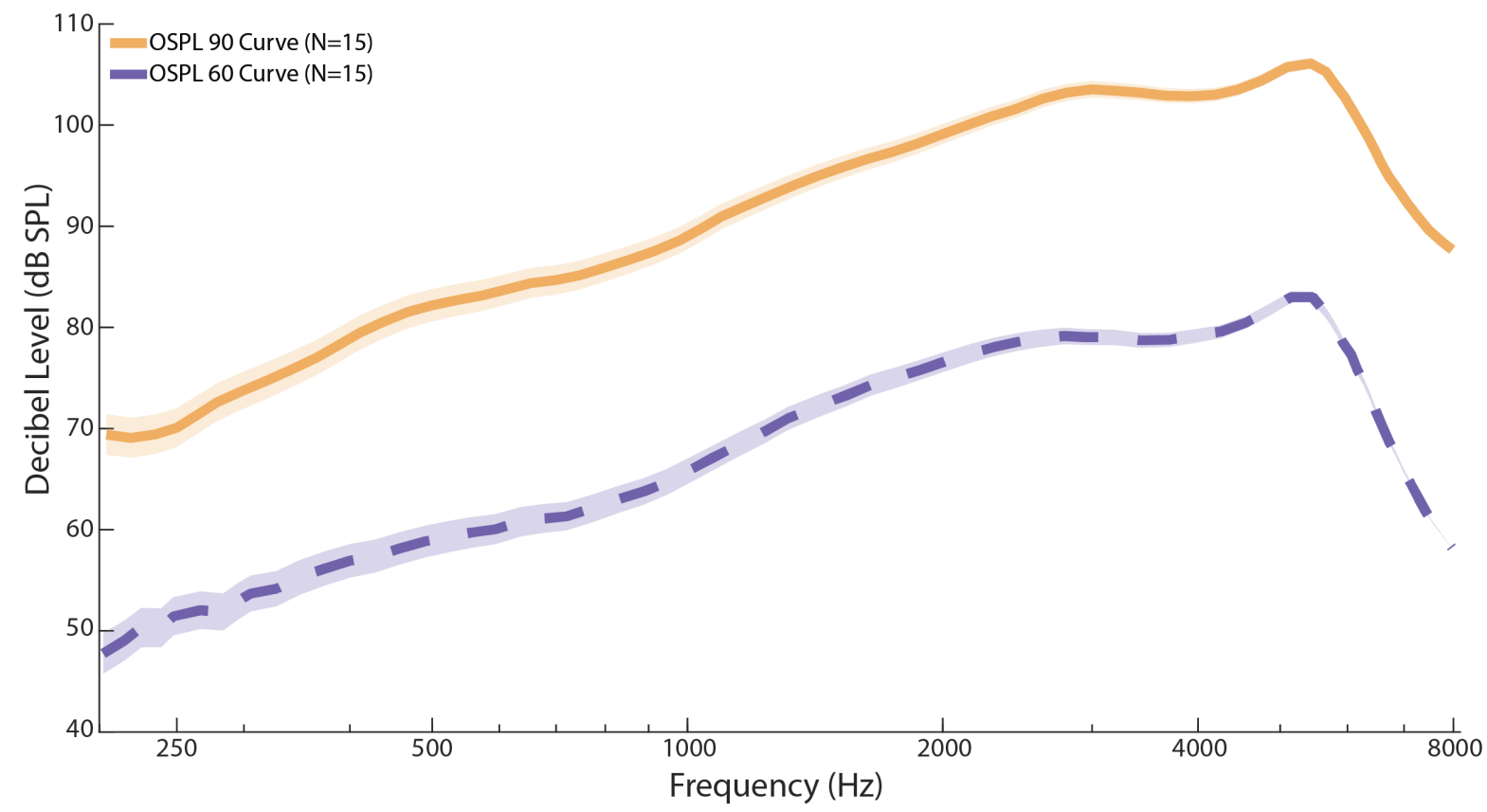

FIG. 2. Electroacoustic Parameter Testing Setup and Results a. A view of the device setup in the test-box. b. This image shows the setup of the device inside the AudioScan Verifit Chamber for testing. The external output of the headphones is placed with putty onto a blue 0.2 cc-coupler which is then attached to the instrument receiver module. c. This shows the placement of the AudioScan speaker output within $1 \mathrm{~mm}$ relative to the microphone input of the LoCHAid.d. The graph shows the OSPL 90 and OSPL 60 curves for the device ( $\mathrm{N}=3$ devices, $\mathrm{n}=5$ trials per device). There is less amplification in the lower frequencies $(<1 \mathrm{kHz})$, and more amplification in the upper frequencies $(>1 \mathrm{kHz})$. 
bioRxiv preprint doi: https://doi.org/10.1101/811828; this version posted October 21, 2019. The copyright holder for this preprint (which was not certified by peer review) is the author/funder, who has granted bioRxiv a license to display the preprint in perpetuity. It is made available under aCC-BY-NC-ND 4.0 International license.

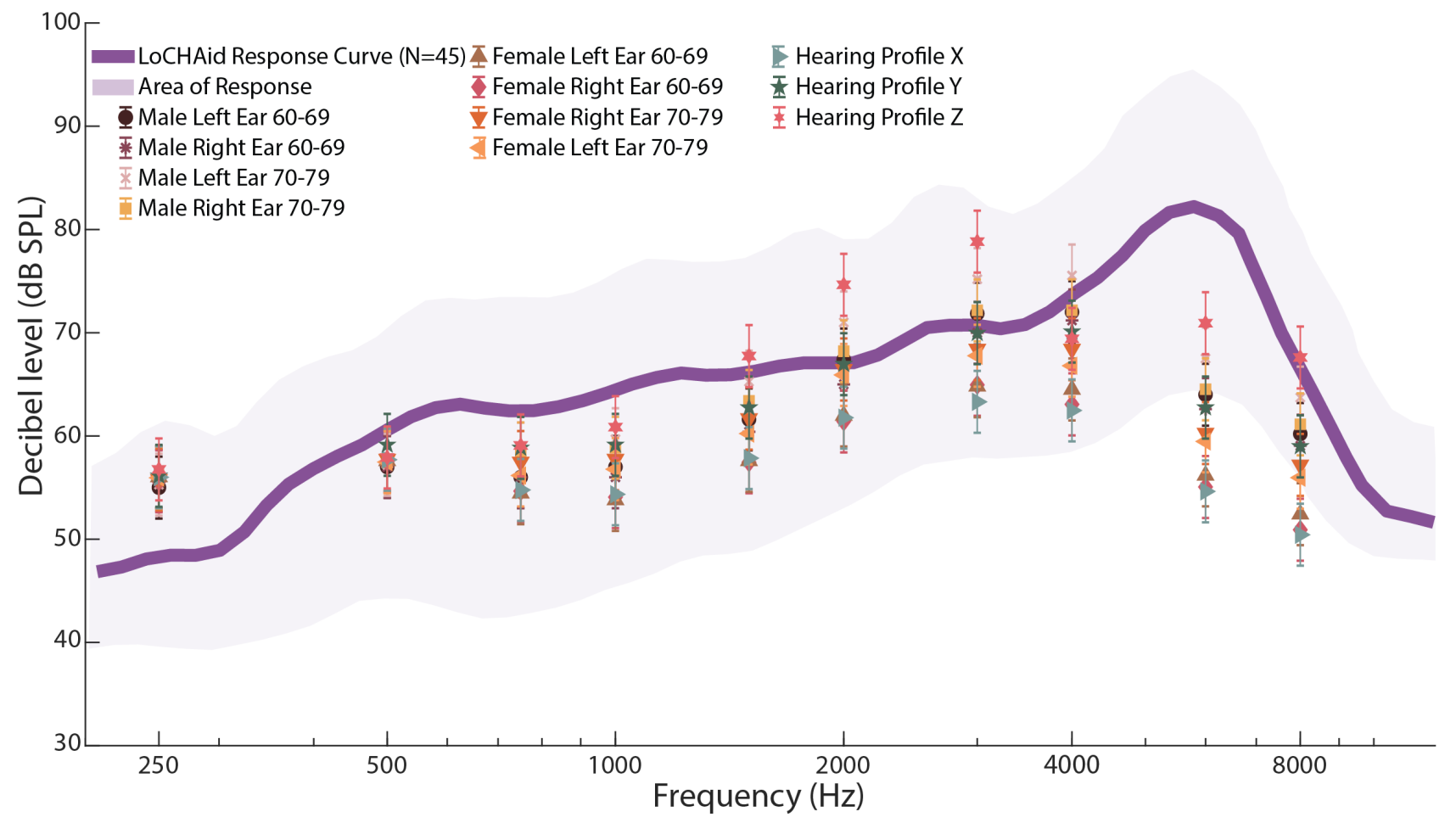

FIG. 3. Audiometric Fitting Results by Speechmap. The graph shows the NA-NL2 targets for 11 profiles. The purple line is the average response of the device on full on gain (no volume reduction) in response to ISTS $65 \mathrm{~dB}$ input; the shaded area of shows the range of response of the device to the input. The targets have a standard error of $3 \mathrm{~dB}$ SPL, which are shown in the error bars. The objective is for the purple line to go through the targets for the device to be fit to the profile. The device well incorporates the range of targets in its area of response, and the average response is well within $10 \mathrm{~dB}$ of the targets except for $6000 \mathrm{~Hz}$. The data is taken from $\mathrm{N}=3$ devices, $\mathrm{n}=15$ trials. 
bioRxiv preprint doi: https://doi.org/10.1101/811828; this version posted October 21,2019 . The copyright holder for this preprint (which was not certified by peer review) is the author/funder, who has granted bioRxiv a license to display the preprint in perpetuity. It is made available under

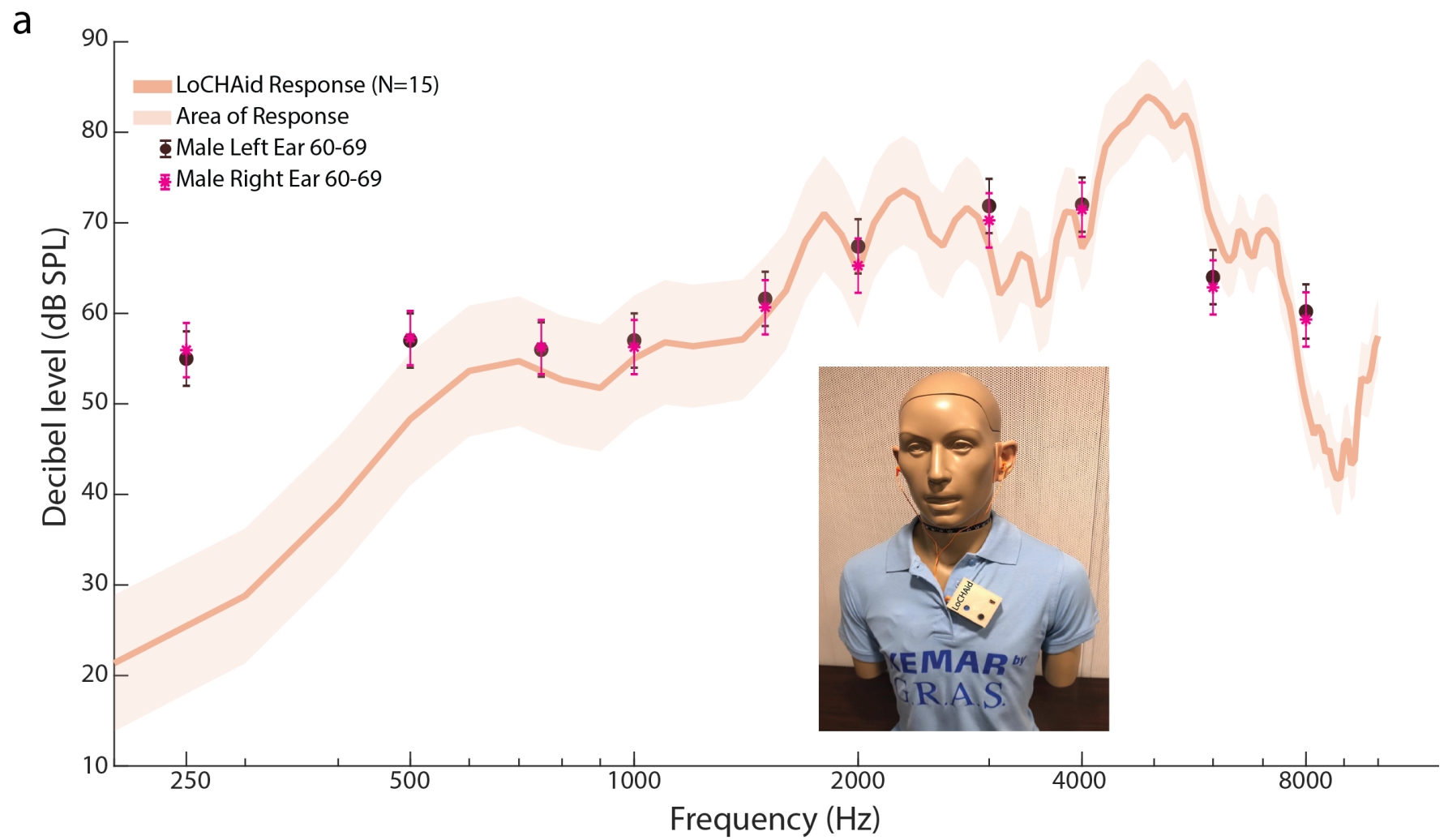

b

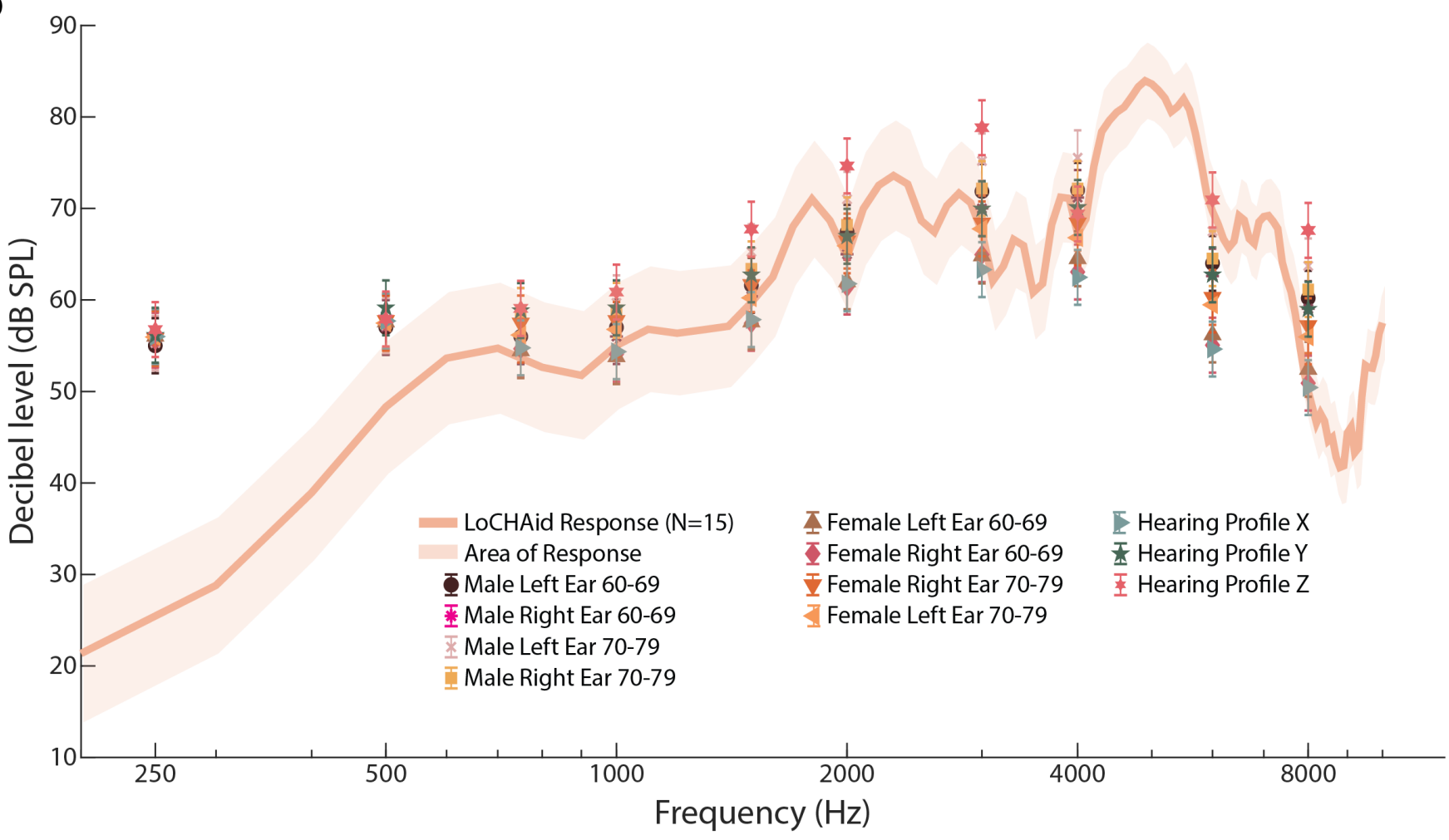

FIG. 4. G.R.A.S KEMAR Audiometric Fitting Results. a.The graph shows the LoCHAid KEMAR Response with NA-NL2 targets from profiles Males 60-69 Left and Right ears. The solid line shows the average response of the KEMAR real ear $(\mathrm{N}=1$ device, $\mathrm{n}=15$ trials); the shaded area represents the standard deviation of the response. The NA-NL2 targets for the profiles from SI Fig S1 are shown as well to see how well the KEMAR response fits the targets. The fits are better than the test-box simulations in Figure 3. Overall, the graph shows that the device very well fits the profiles within $5 \mathrm{~dB}$ SPL, except at lower frequencies. However, at lower frequencies, it is better to have less gain, as there is user complaint of hearing background noise. The inset image shows the setup of the LoCHAid with hearing buds on the G.R.A.S KEMAR Manikin of testing. b. The graph shows the NA-NL2 targets for all 11 profiles with the response of the LoCHAid. The response is better matched to the targets in the KEMAR simulation as compared to the test-box Speechmap simulations, under both Loose and Strict Criteria - see discussion in main text. 
bioRxiv preprint doi: https://doi.org/10.1101/811828; this version posted October 21, 2019. The copyright holder for this preprint (which was not certified by peer review) is the author/funder, who has granted bioRxiv a license to display the preprint in perpetuity. It is made available under aCC-BY-NC-ND 4.0 International license.

\begin{tabular}{|c|c|c|}
\hline Components & Mass Production Cost & \\
\hline Earphones (i) & $\$ 0.04$ & \\
\hline Audio Jack (ii) & $\$ 0.03$ & \\
\hline $2 \times 1000$ pF Capacitor (iii) & $\$ 0.02$ & \\
\hline $2 \times 1 \mathrm{uF}$ Capacitors (iv) & $\$ 0.02$ & \\
\hline $1 \times 15$ uF Capacitor (v) & $\$ 0.01$ & \\
\hline $5 \mathrm{k} \Omega$ Trim Pot Potentiometer (vi) & $\$ 0.06$ & \\
\hline 6 pronged - Slide Switch (vii) & $\$ 0.03$ & \\
\hline Open Source Electret Microphone (viii) & $\$ 0.10$ & \\
\hline Open Source Stereo Class D 3.7 Amplifier (ix) & $\$ 0.48$ & \\
\hline Circuit Board (x) & $\$ 0.05$ & \\
\hline 3D Printed PLA Casing (xi) & $\$ 0.06$ & \\
\hline $2 \times 6.8 \mathrm{k} \Omega$ Resistors (xii) & $\$ 0.02$ & \\
\hline Total Cost Without Batteries & $\$ 0.92$ & Total Cost With Batteries \\
\hline 2 AA Alkaline Batteries and Holder (xiii) & $\$ 0.13$ & $\$ 1.05$ \\
\hline 3 V Coin Cell Battery and Holder (xiv) & $\$ 0.06$ & $\$ 0.98$ \\
\hline
\end{tabular}

TABLE I. Component Costs of the LoCHAid. The table lists the costs for acquiring individual components in bulk of 10000 pieces. The LoCHAid is assumed has been created from the following: (i) a set of earphones (ModelGF-923, In-Ear, 3.5mm Connector, from Boluo Golden Fortune Electronic Manufacture Factory, wwww.alibaba.com, P/N 60249739970), (ii) a audio jack (1/4" 3.5mm PCB Mount Female Socket 5 pin, from Yueqing Daier Electron Co. LTD, from www.alibaba.com, M/N EJ-214M); (iii) $21000 \mathrm{pF}$ capacitors (SMD/SMT 1000 pF 50V Multilayer Ceramic Capacitor, from Part Rescue Technology, from www.alibaba.com, M/N VJ0603Y102KXACW1BC); (iv) $21 \mathrm{uF}$ capacitors (SMD Ceramic Capacitor 1uF 50 V, from Shenzhen Yuzens Technologies Co. Ltd, from www.alibaba.com, M/N CL10A105KB8NNC); (v) a 15uF capacitor (250V 450vac $15 \mathrm{uF}$ polyester capacitor, from Shenzhen Weitaixu Cpacitors Co., Ltd., from www.alibaba.com, M/N cbb61 $15 \mathrm{uF}$ run capacitor); (vi) a $5 \mathrm{k} \Omega$ Trim Pot Potentiometer (Cermaic Bourns Variable Resistor, from Changhoo Kennon Electronics Co. Ltd., from www.alibaba.com, M/N 3006P); (vii) a 6 pronged slide switch (Mini Slide switch, from A-Key Electronics Technology, from www.alibaba.com, M/N MSS-22D16); (viii) an electret microphone module (Utilising MAX9814, from Shenzhen Ronghai Electronics Co. Ltd, from www.alibaba.com, M/N MAX9814); (ix) a stereo 3.7 W amplifier (MAX98306 Stereo 3.7W Class D Amplifier, P/N MAX98306ETD+, from www.maximintegrated.com. P/N MAX98306); (x) a circuit board (Prototyping Universal Board PCB Double Sided 4 x 6 cm board, from Shenzhen Androw Technology Limited, D/C YC045-53, www.alibaba.com $\mathrm{P} / \mathrm{N}$ 60529535100); (xi) 3D printed PLA casing is obtained in bulk (PLA plastic granules for 3D filament 3D material PLA plastic pellet, from Yasin, Guangdong China, from www.alibaba.com, M/N PLA pellets, JSC-310); (xii) 26.8 k $\Omega$ Resistors (Resistors $0.4 \mathrm{~W} 6.8 \mathrm{k} \Omega$, from Shanhai Group Limited, from www.alibaba.com, M/N MMA02040C6801FB300). The LoCHAid can be powered by several types of batteries as long they deliver 3V; here, we present two forms - (xiii) 2 AA batteries (Entop 1.5V AA Carbon Zinc, from Suzhou South Large Batter Co., Ltd., www.alibaba.com P/N 60643508502) which needs a battery holder (2 AA 1.5V Battery Holder, from Yueqing Daier Electronics Co., Ltd., from www.alibaba.com, M/N BH5-2003); (xiv) or a coin cell battery (3V Lithium Button Cell, from Shenzhen Gmcell Technology Co., Ltd. P/N CR2032, www.alibaba.com, P/N 60251728326) which needs a coin cell holder (\$0.03 (Black 3V Coin Button Holder, Yueqing Daier Electronics Co., Ltd., from www.alibaba.com, M/N BH2032-3). *All links and prices last accessed September 17, 2019.

\begin{tabular}{|c|c|c|c|c|}
\hline Electroacoustic Parameter & WHO Recommendation & ANSI/CTA-2051 & LoCHAid & Met \\
\hline Max OSPL 90 & 100-130 dB SPL & $<120 \mathrm{~dB}$ SPL & $107 \mathrm{~dB}$ SPL & Yes \\
\hline OSPL $90 @ 1 \mathrm{kHz}$ & 90-124 dB SPL & NS & $90 \mathrm{~dB}$ SPL & Yes \\
\hline Average OSPL 90 & NS & NS & $96 \mathrm{~dB}$ SPL & \\
\hline Average Gain & NS & NS & $15 \mathrm{~dB}$ SPL & \\
\hline Total Harmonic Distortion & $500 \mathrm{~Hz}<8 \%$ & & $500 \mathrm{~Hz}=1 \%$ & \\
\hline 1otal Harmonc Distortion & $1000 \mathrm{~Hz}<8 \%$ & $500 \mathrm{~Hz}<5 \%$ & $1000 \mathrm{~Hz}=1 \%$ & Yes \\
\hline & $1600 \mathrm{~Hz}<2 \%$ & & $1600 \mathrm{~Hz}=1 \%$ & \\
\hline Equivalent Input Noise & $<30 \mathrm{~dB}$ SPL & $<32$ dB SPL & $40 \mathrm{~dB}$ SPL & No \\
\hline Range of Response and & $200-8000 \mathrm{~Hz}$ & $250-5000 \mathrm{~Hz}$ & $<200->8000 \mathrm{HZ}$ & Yes \\
\hline Smoothness & Smoothness - NS & Smoothness - No sharp peaks & & Smooth \\
\hline Battery Life & 2-3 Weeks & NS & $\begin{array}{c}20 \text { days } \\
\text { (with } 2 \text { AA batteries) }\end{array}$ & Yes \\
\hline HFA $(1,1.5,3 \mathrm{kHz}) @$ OSPL 90 & NS & NS & $93 \mathrm{~dB}$ SPL & \\
\hline $\operatorname{HFA}(4,5,6 \mathrm{kHz}) @$ OSPL 90 & NS & NS & $103 \mathrm{~dB}$ SPL & \\
\hline
\end{tabular}

TABLE II. Electroacoustic Parameter Results and Comparison. The table lists the ANSI Parameters (OSPL 90, OSPL 60, Total Harmonic Distortion, High Frequency Average, Average Gain, Max OSPL 90) that were tested on the LoCHAid, the WHO Recommendations PPP for the device, the ANSI/CTA-2051 recommendations, and the results from the LoCHAID, and whether the targets were met or not for both sets of recommendations $[12,26]$. The device is able to meet all the targets except for Equivalent Input Noise. See discussion in main text about EIN. ${ }^{*} N S=$ Not Specified 